\title{
The Quantum Bang Hypothesis: An Alternative to Dark Matter and Dark Energy
}

\author{
Patrick G. Tonin \\ Saint Martin de Londres, France \\ Email:pgtonin@gmail.com
}

How to cite this paper: Tonin, P.G. (2020) The Quantum Bang Hypothesis: An Alternative to Dark Matter and Dark Energy. Journal of High Energy Physics, Gravita tion and Cosmology, 6, 753-764.

https://doi.org/10.4236/jhepgc.2020.64050

Received: September 2, 2020

Accepted: October 16, 2020

Published: October 19, 2020

Copyright $\odot 2020$ by author(s) and Scientific Research Publishing Inc. This work is licensed under the Creative Commons Attribution International License (CC BY 4.0).

http://creativecommons.org/licenses/by/4.0/

\begin{abstract}
We hypothesize that the quantum realm and the cosmos are linked by a scaling relation where the gravitational coupling constant $\alpha_{G}$ is the scale factor and decreases with cosmic time. We propose a simple cosmological model where cosmic inflation, dark energy and dark matter could be redundant concepts. We show that cosmological parameters such as the Hubble constant, the age, density and mass of the observable Universe could be derived simply from quantum parameters. Finally, we propose a fundamental MOND formula with no interpolating function and an acceleration parameter simply derived from the Hubble constant.
\end{abstract}

\section{Keywords}

Dark Matter, Dark Energy, Inflation, Cosmological Parameters, MOND

\section{Introduction}

The $\Lambda$ CDM (Lambda Cold Dark Matter) cosmological model provides a widely accepted description of the Universe. However, the fundamental nature of the $\Lambda \mathrm{CDM}$ main components, namely cosmic inflation, dark matter and dark energy remains a mystery [1] [2]. In this paper, we propose a cosmological model where these components might not be required. Our proposal is built upon the hypothesis that the gravitational coupling constant $\alpha_{G}$ acts as a scale factor between the quantum realm and the cosmos and decreases with cosmic time. We introduce a simple correlation between the electron Compton time and the age of the Universe, between the electron Compton wavelength and the Hubble radius, and between the electron mass and the mass of the observable Universe. These correlations suggest that the Hubble parameter will always equal the inverse of the age of the Universe and that the Universe density will always equal 
the Universe critical density. It is predicted that the mass and size of particles decrease with cosmic time, while the mass and size of the observable Universe increase with cosmic time. The Newtonian gravitational constant $G$ and the Planck constant $h$ are also predicted to decrease with cosmic time. According to our proposal, the measured acceleration of the Universe expansion is an illusion induced by the gravitational acceleration of the Universe, this same acceleration is also responsible for the discrepancy between the expected and observed velocities of stars in galaxies. We propose a modified Newtonian equation for the velocity of stars in a galaxy, which would eliminate the need for dark matter. Overall, this proposal describes a Universe where all constituents, including ourselves, are constantly changing scale (in addition to the observed Hubble expansion), but this change in scale remains unnoticed as all our surroundings, including our units of measurement and most physical constants are also changing scale with cosmic time.

\section{The Age, Hubble Radius and Mass of the Universe}

We hypothesize that the quantum realm and the cosmos are linked by a scale relation. We propose the following three novel equations from which our cosmological model is deduced. These equations are valid for all cosmic times, as explained in Section 5. The values used in this paper are taken from CODATA 2018 [3].

The age of the Universe $t_{0}$ is linked to the electron Compton time:

$$
t_{0}=\frac{t_{e}}{\pi \alpha_{G}}=\frac{2 \mu t_{P}}{\sqrt{\alpha_{G}^{3}}}=4.3618 \times 10^{17} \mathrm{~s}(13.822 \mathrm{Gyr})
$$

where $t_{e}$ is the electron Compton time $\left(t_{e}=\lambda_{e} / c\right), \alpha_{G}$ is the gravitational coupling constant defined using a pair of protons $\left(\alpha_{G}=\left(m_{p r} / m_{p}\right)^{2}\right), \mu$ is the proton to electron mass ratio and $t_{P}$ is the Planck time.

This value agrees with the Planck Collaboration 2018 estimate [4] of $t_{0}=$ $13.801 \pm 0.024 \mathrm{Gyr}$

The Hubble radius $R_{h}$ is linked to the electron Compton wavelength:

$$
R_{h}=\frac{\lambda_{e}}{\pi \alpha_{G}}=\frac{2 \mu l_{P}}{\sqrt{\alpha_{G}^{3}}}=1.3076 \times 10^{26} \mathrm{~m}
$$

where $\lambda_{e}$ is the electron Compton wavelength, $\alpha_{G}$ is the gravitational coupling constant defined using a pair of protons, $\mu$ is the proton to electron mass ratio and $l_{P}$ is the Planck length.

The mass of the observable Universe $M_{u}$ is linked to the electron mass:

$$
M_{u}=\frac{\mu^{2} m_{e}}{\alpha_{G}^{2}}=\frac{\mu m_{P}}{\sqrt{\alpha_{G}^{3}}}=8.8043 \times 10^{52} \mathrm{~kg}
$$

where $\mu$ is the proton to electron mass ratio, $m_{e}$ is the electron mass, $\alpha_{G}$ is the gravitational coupling constant defined using a pair of protons, and $m_{P}$ is the Planck mass. 
From Equations (1), (2) and (3), we deduce further equations and 12 key predictions which are presented in the following sections.

\section{The Hubble Constant}

From Equations (1) and (2), we deduce the following equation for the Hubble constant $H_{0}$ :

$$
H_{0}=\frac{c}{R_{h}}=\frac{\pi \alpha_{G}}{t_{e}}=\frac{\sqrt{\alpha_{G}^{3}}}{2 \mu t_{P}}=\frac{1}{t_{0}}=70.74 \mathrm{~km} \cdot \mathrm{s}^{-1} \cdot \mathrm{Mpc}^{-1}
$$

where $c$ is the speed of light, $R_{h}$ is the Hubble radius, $t_{e}$ is the electron Compton time, $\alpha_{G}$ is the gravitational coupling constant defined using a pair of protons, $\mu$ is the proton to electron mass ratio, $t_{P}$ is the Planck time and $t_{0}$ is the age of the Universe.

This value falls between the Planck Collaboration 2018 estimate [4] of $H_{0}=$ $67.37 \pm 0.54 \mathrm{~km} \cdot \mathrm{s}^{-1} \cdot \mathrm{Mpc}^{-1}$ and the distance-ladder estimate from the SHOES project 2019 [5] of $H_{0}=74.03 \pm 1.42 \mathrm{~km} \cdot \mathrm{s}^{-1} \cdot \mathrm{Mpc}^{-1}$.

Prediction 1: The Hubble constant will always equal the inverse of the age of the Universe.

We note that this statement is in agreement with the $R_{h}=c t$ model proposed by Melia Shevchuk [6].

\section{The Universe Density}

From Equations (1), (2) and (3), we deduce the following equation for the mass of the observable Universe $M_{u}$ :

$$
M_{u}=\frac{R_{h} c^{2}}{2 G}=\frac{t_{0} c^{3}}{2 G}=\frac{c^{3}}{2 G H_{0}}=8.8043 \times 10^{52} \mathrm{~kg}
$$

where $R_{h}$ is the Hubble radius, $c$ is the speed of light, $G$ is the Newtonian gravitational constant, $t_{0}$ is the age of the Universe and $H_{0}$ is the Hubble constant.

The Universe Hubble volume is defined as:

$$
V_{h}=\frac{4 \pi R_{h}^{3}}{3}=9.3662 \times 10^{78} \mathrm{~m}^{3}
$$

Using Equations (5) and (6), and considering that the observable Universe volume is equal to the Hubble volume (as per our hypothesis), we propose the following equation for the Universe density $\rho_{u}$ :

$$
\rho_{u}=\frac{M_{u}}{V_{h}}=\frac{3 c^{3}}{8 \pi G H_{0} R_{h}^{3}}=\frac{3 H_{0}^{2}}{8 \pi G}=9.400 \times 10^{-27} \mathrm{~kg} \cdot \mathrm{m}^{-3}
$$

This equation matches the equation for the critical density of a Friedmann-Robertson-Walker (FRW) universe.

Prediction 2: The Universe density will always equal the Universe critical density.

\section{The Fundamental Units of Time, Length and Mass}

In order for Equation (1) to be valid as the Universe ages, either the gravitational 
coupling constant $\alpha_{G}$ or the electron Compton time $t_{\odot}$ or both, have to vary. We propose that the age of the Universe and the electron Compton time are respectively scaled up and scaled down values of a fundamental unit of time (that we will call $T_{0}$ ), the scale factor is represented by the square root of the gravitational coupling constant. This unit of time represents a fundamental reference and is invariant under the scale transformation.

The same principle is applied to Equations (2) and (3) to obtain the fundamental unit of length $L_{0}$ and the fundamental unit of mass $M_{0}$.

In relation to these units, the Universe expands and gains mass while the elementary particles shrink and lose mass.

The fundamental unit of time $T_{0}$ is defined as:

$$
T_{0}=t_{0} \pi \sqrt{\alpha_{G}}=\frac{t_{e}}{\sqrt{\alpha_{G}}}=\frac{2 \pi \mu t_{P}}{\alpha_{G}}=0.10531 \mathrm{~s}
$$

While the age of the Universe $t_{0}$ increases, the gravitational coupling constant $\alpha_{G}$ decreases. While the gravitational coupling constant $\alpha_{G}$ decreases, the electron Compton time $t_{e}$ decreases.

From Equation (8), we deduce how the gravitational coupling constant $\alpha_{G}$ varies with cosmic time $t$.

$$
\alpha_{G}(t)=\frac{T_{0}^{2}}{\pi^{2} t^{2}} \propto t^{-2}
$$

From Equation (8), we deduce how the electron Compton time $t_{e}$ varies with cosmic time $t$ :

$$
t_{e}(t)=\frac{T_{0}^{2}}{\pi t} \propto t^{-1}
$$

The fundamental unit of length $L_{0}$ is defined as:

$$
L_{0}=R_{h} \pi \sqrt{\alpha_{G}}=\frac{\lambda_{e}}{\sqrt{\alpha_{G}}}=\frac{2 \pi \mu l_{P}}{\alpha_{G}}=3.15714 \times 10^{7} \mathrm{~m}
$$

While the Hubble radius $R_{h}$ increases, the gravitational coupling constant $\alpha_{G}$ decreases.

While the gravitational coupling constant $\alpha_{G}$ decreases, the electron Compton wavelength $\lambda_{e}$ decreases.

From Equations (8) and (11), we deduce how the Hubble radius $R_{h}$ varies with cosmic time $t$ :

$$
R_{h}(t)=\frac{L_{0} t}{T_{0}} \propto t
$$

From Equations (8) and (11) we deduce how the electron Compton wavelength $\lambda_{e}$ varies with cosmic time $t$.

$$
\lambda_{e}(t)=\frac{L_{0} T_{0}}{\pi t} \propto t^{-1}
$$

The fundamental unit of mass $M_{0}$ is defined as: 


$$
M_{0}=\frac{M_{u}}{\mu^{2}} \alpha_{G}=\frac{m_{e}}{\alpha_{G}}=\frac{m_{P}}{\mu \sqrt{\alpha_{G}}}=15.4235 \times 10^{7} \mathrm{~kg}
$$

While the mass of the observable Universe $M_{u}$ increases, the gravitational coupling constant $\alpha_{G}$ decreases. While the gravitational coupling constant $\alpha_{G}$ decreases, the electron mass $m_{e}$ decreases.

From Equations (8) and (14), we deduce how the mass of the observable Universe $M_{u}$ varies with cosmic time $t$.

$$
M_{u}(t)=\frac{\pi^{2} \mu^{2} M_{0} t^{2}}{T_{0}^{2}} \propto t^{2}
$$

From Equations (8) and (14), we deduce how the electron mass $m_{e}$ varies with cosmic time $t$ :

$$
m_{e}(t)=\frac{M_{0} T_{0}^{2}}{\pi^{2} t^{2}} \propto t^{-2}
$$

Prediction 3: The mass and size of the Universe increase with cosmic time. Prediction 4: The mass and size of particles decrease with cosmic time.

We note that in our hypothesis, the speed of light $c$ remains constant over cosmic time:

$$
c=\frac{L_{0}}{T_{0}}=299792458 \mathrm{~m} \cdot \mathrm{s}^{-1}
$$

\section{The Newtonian Gravitational Constant $G$}

From Equations (5) and (15), we deduce how the Newtonian gravitational constant $G$ varies with cosmic time $t$ :

$$
G(t)=\frac{L_{0}^{3}}{2 \pi^{2} \mu^{2} T_{0} M_{0} t} \propto t^{-1}
$$

where $T_{0}, L_{0}$ and $M_{0}$ are the fundamental units of time, length and mass as defined in Equations (8), (11) and (14), and $\mu$ is the proton to electron mass ratio.

Prediction 5: The Newtonian gravitational constant $G$ decreases with cosmic time as $G \propto t^{-1}$.

We note that this statement is in agreement with Dirac's variable $G$ hypothesis [7].

\section{The Planck Constant $h$}

From Equations (10) and (16), we deduce how the Planck constant $h$ varies with cosmic time $t$ :

$$
h(t)=\frac{M_{0} L_{0}^{2} T_{0}^{2}}{\pi^{3} t^{3}} \propto t^{-3}
$$

where $T_{0}, L_{0}$ and $M_{0}$ are the fundamental units of time, length and mass as defined in Equations (8), (11) and (14), and $\mu$ is the proton to electron mass ratio.

Prediction 6: The Planck constant $h$ decreases with cosmic time as $h \propto t^{-3}$. 


\section{The Universe Density Time Variation}

From Equations (4), (7) and (15), we deduce how the Universe density $\rho_{u}$ varies with cosmic time $t$.

$$
\rho_{u}(t)=\frac{3 \pi \mu^{2} T_{0} M_{0}}{4 L_{0}^{3} t} \propto t^{-1}
$$

where $T_{0}, L_{0}$ and $M_{0}$ are the fundamental units of time, length and mass as defined in Equations (8), (11) and (14), and $\mu$ is the proton to electron mass ratio.

Prediction 7: The Universe density $\rho_{u}$ decreases with cosmic time as $\rho_{u} \propto t^{-1}$.

\section{The Number of Protons in the Observable Universe}

Using Equation (3), we can estimate the number of protons in the observable Universe as follows:

$$
N_{p r}=\frac{M_{u}}{m_{p r}}=\frac{\mu}{\alpha_{G}^{2}}=5.2637 \times 10^{79}
$$

where $M_{u}$ is the mass of the observable Universe, $m_{p r}$ is the proton mass, $\mu$ is the proton to electron mass ratio and $\alpha_{G}$ is the gravitational coupling constant defined using a pair of protons.

From Equations (15) and (16), we deduce how the number of protons in the observable Universe varies with cosmic time $t$.

$$
N_{p r}(t)=\frac{\mu \pi^{4} t^{4}}{T_{0}^{4}} \propto t^{4}
$$

where $T_{0}, L_{0}$ and $M_{0}$ are the fundamental units of time, length and mass as defined in Equations (8), (11) and (14), and $\mu$ is the proton to electron mass ratio.

Prediction 8: The number of protons in the Universe increases with cosmic time as $N_{p r} \propto t^{4}$.

The continuous matter creation predicted by our hypothesis could be considered to violate the law of conservation of energy. However, this would not be the case if it was counterbalanced by a continuous creation of negative gravitational potential energy. This idea has been previously proposed by Jordan [8].

\section{The Acceleration of the Universe Expansion}

In a Friedmann-Robertson-Walker (FRW) cosmology, the time derivative of the Hubble parameter $\dot{H}$ can be written in terms of the deceleration parameter $q$ :

$$
\dot{H}=-H^{2}(1+q)
$$

where $H$ is the Hubble parameter and $q$ is the deceleration parameter.

From Equation (4), we deduce the following equation for the time derivative of the Hubble parameter $\dot{H}$ :

$$
\dot{H}=-\frac{1}{t^{2}}=-H^{2}
$$


where $t$ is the cosmic time and $H$ is the Hubble parameter.

Joining Equations (23) and (24), we obtain:

$$
\dot{H}=-H^{2}(1+q)=-H^{2}
$$

From Equation (25), we deduce that the parameter $q$ will always equal zero and therefore the acceleration of the Universe expansion is predicted to be null at all times.

However, the acceleration of the Universe expansion has been confirmed by different measurements [9] [10] [11] [12]. We propose that the measured acceleration of the Universe expansion is merely an illusion induced by the gravitational acceleration of the Universe.

We define the Universe gravitational acceleration $g_{u}$ as:

$$
g_{u}=\frac{G M_{u}}{R_{h}^{2}}=\frac{c}{2 t}=\frac{c H}{2}
$$

where $G$ is the Newtonian gravitational constant, $M_{u}$ is the mass of the observable Universe, $R_{h}$ is the Hubble radius, $c$ is the speed of light, $t$ is the cosmic time and $H$ is the Hubble parameter.

We define the Universe gravitational acceleration per unit of length $a_{u}$ as:

$$
a_{u}=\frac{g_{u}}{R_{h}}=\frac{1}{2 t^{2}}=\frac{H^{2}}{2}
$$

In an FRW cosmology, the acceleration of the Universe expansion $U_{\text {acc }}$ is defined as:

$$
U_{a c c}=-H^{2}(q)
$$

where $H$ is the Hubble parameter and $q$ is the deceleration parameter.

We propose that the measured acceleration of the Universe expansion will have exactly the same value as the gravitational acceleration of the Universe.

Joining Equations (27) and (28) we obtain:

$$
a_{u}=U_{a c c}=-H^{2}(q)=\frac{H^{2}}{2}
$$

From Equation (29), we deduce that the measured value of the deceleration parameter $q$ will always be -0.5 .

We note that this value is in line with the currently accepted value of -0.55 of the $\Lambda$ CDM model.

Prediction 9: The measured acceleration of the Universe expansion is an illusion induced by the Universe gravitational acceleration, the real acceleration is null.

Prediction 10: The measured acceleration of the Universe expansion will have exactly the same value as the gravitational acceleration of the Universe.

The value of $q$ being fixed at -0.5 implies that the measured deceleration of the Hubble parameter is also affected by the Universe gravitational acceleration.

In an FRW cosmology, the deceleration of the Hubble parameter is defined as: 


$$
H_{d e c}=H^{2}(1+q)
$$

If the deceleration parameter $q$ is always fixed at -0.5 (as implied by Equation (29)), then the measured acceleration of the Universe expansion will always equal the measured deceleration of the Hubble parameter, and both will always equal the Universe gravitational acceleration.

Joining Equations (29) and (30), we obtain:

$$
-H^{2}(q)=H^{2}(1+q)=\frac{H^{2}}{2}
$$

Prediction 11: The measured acceleration of the Universe expansion will always equal the measured deceleration of the Hubble parameter.

\section{A Fundamental MOND Formula}

The velocities of stars in galaxies are observed to be larger than expected based on Newtonian mechanics [13]. This has led scientists to invoke the presence of dark matter in the Universe. We propose that this discrepancy is induced by the Universe gravitational acceleration.

From Equation (26), we calculate the Universe gravitational acceleration $g_{u}$ :

$$
g_{u}=\frac{G M_{u}}{R_{h}^{2}}=\frac{c}{2 t_{0}}=\frac{c H_{0}}{2}=3.4365 \times 10^{-10} \mathrm{~m} \cdot \mathrm{s}^{-2}
$$

where $G$ is the Newtonian gravitational constant, $M_{u}$ is the mass of the observable Universe, $R_{h}$ is the Hubble radius, $c$ is the speed of light, $t_{0}$ is the cosmic time and $H_{0}$ is the Hubble parameter.

Taking into account the effect of the Universe gravitational acceleration, we propose the following equation for a star rotation velocity $v$.

$$
v=\sqrt{\frac{G M}{r}+\sqrt{G M g_{u}}}=\sqrt{\frac{G M}{r}+\sqrt{\frac{G M c H_{0}}{2}}}
$$

where $G$ is the Newtonian gravitational constant, $M$ is the mass of the galaxy, $r$ is the distance of the star from the center of the galaxy, $g_{u}$ is the Universe gravitational acceleration, $c$ is the speed of light and $H_{0}$ is the Hubble constant.

We observe that for large values of $r$, the equation could take the following simplified form:

$$
v^{4}=G M g_{u}
$$

We note the similarity with Milgrom MOND's formula [14].

Prediction 12: The rotation curve of galaxies is influenced by the Universe gravitational acceleration.

\section{The Weinberg Formula}

In his book on Gravitation and Cosmology, Weinberg [15] presented an enigmatic empirical formula linking the mass of an elementary particle, such as a pion, to the fundamental constants $G, \hbar, c$ and the Hubble constant $H_{0}$. To this day, it is not known if this formula is just a coincidence or has an underlying 
fundamental significance. We propose that this formula is not a coincidence.

The Weinberg formula is:

$$
m_{\pi} \approx\left(\frac{\hbar^{2} H_{0}}{G c}\right)^{\frac{1}{3}}
$$

where $m_{\pi}$ is the mass of a pion, $\hbar$ is the reduced Planck constant, $H_{0}$ is the Hubble constant, $G$ is the Newtonian gravitational constant and $c$ is the speed of light.

Equation (4) can be reformulated as:

$$
H_{0}=\frac{\pi \alpha_{G}}{t_{e}}=\frac{\mu^{2} m_{e}^{3} G c}{2 \hbar^{2}}
$$

where $\alpha_{G}$ is the gravitational coupling constant defined using a pair of protons, $t_{e}$ is the electron Compton time, $\mu$ is the proton to electron mass ratio, $m_{e}$ is the electron mass, $G$ is the Newtonian gravitational constant, $c$ is the speed of light and $\hbar$ is the reduced Planck constant.

Extracting the electron mass $m_{e}$ and using the value of $H_{0}$ in Equation (4), we obtain the exact value for the electron mass $m_{e}$ (CODATA 2018 [3]):

$$
m_{e}=\left(\frac{2 \hbar^{2} H_{0}}{\mu^{2} G c}\right)^{\frac{1}{3}}=9.1093837 \times 10^{-31} \mathrm{~kg}
$$

The equation is very similar to the Weinberg formula. It is also valid for any cosmic time as the values of $m_{\mathcal{e}} G$ and $h$ vary as per Equations (16), (18) and (19).

\section{Discussion}

In this paper, we have proposed a cosmological model based on the hypothesis that the quantum realm and the cosmos are linked by a scaling relation. This idea was first proposed by Dirac [7] and is known as the Large Numbers Hypothesis (LNH). The LNH has generated considerable interest [16] and, although it has not been recognized as a viable model, the possible variability of physical constants has not yet been completely ruled out. Unlike the LNH and other propositions with variable physical constants, our approach introduces a very precise relationship between the quantum realm and the cosmos via a set of equations for values such as the Hubble constant, the age and density of the Universe and the measured acceleration of the Universe expansion. The predicted values are in agreement with the currently accepted values and are obtained without any ad hoc parameters. From Equations (20) and (22), we deduce that the Universe started with a finite density and possibly from just one single atom, and from Equations (4) and (7), we deduce that the Universe has never expanded faster than the speed of light and has always been flat, thus removing the need to invoke a period of cosmic inflation [17] to explain the horizon problem [18] and the flatness of the Universe [19]. Our proposal predicts that the acceleration of the Universe expansion is an illusion induced by the gravitational acce- 
leration of the Universe, this in turn would remove the need to invoke dark energy [20] to explain the acceleration of the Universe expansion. The same gravitational acceleration is also predicted to increase the velocities of stars in galaxies. Consequently, we have proposed a modified Newtonian equation which, if proved correct, would remove the need to invoke dark matter [21] to explain the dynamics of galaxies and clusters. We note that the problems of dark matter and dark energy could, in principle, also be solved through extended theories of gravity [22]. Our hypothesis predicts a change in scale not only at the cosmological level but also at the quantum level. Although the cosmological scale expansion can easily be confirmed through the Hubble expansion, it is unclear how a quantum scale contraction would be perceived by a human being living in such a universe. We will need to evaluate the possibility of measuring such a change in physical constants if our units of measurement are also changing scale with cosmic time. This question has been raised before with conflicting views [23] [24] and the answer remains uncertain.

\section{Conclusion}

We have shown that from three simple equations linking the quantum realm and the cosmos, it is possible to build a simple and coherent model of the Universe, where cosmological parameters could be derived simply from quantum parameters. Along with removing the need to invoke components like inflation, dark matter and dark energy to explain cosmological observations, our proposal could provide the basis for a much simpler cosmological model without any ad hoc parameters.

\section{Conflicts of Interest}

The author declares no conflicts of interest regarding the publication of this paper.

\section{References}

[1] Steinhardt, P.J. (2011) The Inflation Debate. Scientific American, 304, 36-43. https://doi.org/10.1038/scientificamerican0411-36

[2] Arun, K., Gudennavar, S. and Sivaram, C. (2017) Dark Matter, Dark Energy, and Alternate Models: A Review. Advances in Space Research, 60, 166-186. https://doi.org/10.1016/j.asr.2017.03.043

[3] Tiesinga, E., Mohr, P.J., Newell, D.B., Barry, N. and Taylor, B.N. (2020) The 2018 CODATA Recommended Values of the Fundamental Physical Constants (Web Version 8.1). http://physics.nist.gov/constants

[4] Aghanim, N., et al. (2018) Planck 2018 Results. VI. Cosmological Parameters. https://arXiv.org/abs/1807.06209

[5] Riess, A.G., Casertano, S., Yuan, W., Macri, L.M. and Scolnic, D. (2019) Large Magellanic Cloud Cepheid Standards Provide a 1\% Foundation for the Determination of the Hubble Constant and Stronger Evidence for Physics beyond $\Lambda \mathrm{CDM}$. The Astrophysical Journal, 876, 85. https://doi.org/10.3847/1538-4357/ab1422 
[6] Melia, F. and Shevchuk, A.S.H. (2012) The $R_{\mathrm{h}}=c t$ Universe. Monthly Notices of the Royal Astronomical Society, 419, 2579-2586. https://doi.org/10.1111/j.1365-2966.2011.19906.x

[7] Dirac, P.A.M. (1937) The Cosmological Constants. Nature, 139, 323. https://doi.org/10.1038/139323a0

[8] Jordan, P.J. (1949) Formation of the Stars and Development of the Universe. Nature, 164, 637-640. https://doi.org/10.1038/164637a0

[9] Riess, A.G., et al. (1998) Observational Evidence from Supernovae for an Accelerating Universe and a Cosmological Constant. The Astronomical Journal, 116, 1009-1038. https://doi.org/10.1086/300499

[10] Perlmutter, S., et al. (1999) Measurements of Omega and Lambda from 42 High Redshift Supernovae. The Astrophysical Journal, 517, 565-586. https://doi.org/10.1086/307221

[11] Eisenstein, D.J., et al. (2005) Detection of the Baryon Acoustic Peak in the Large-Scale Correlation Function of SDSS Luminous Red Galaxies. The Astrophysical Journal, 633, 560-574. https://doi.org/10.1016/j.crhy.2012.04.009

[12] Astier, P. and Pain, R. (2012) Observational Evidence of the Accelerated Expansion of the Universe. Comptes. Rendus Physiques, 13, 521-538. https://doi.org/10.1086/182804

[13] Rubin, V.C., Thonnard, N. and Ford, W.K. (1978) Extended Rotation Curves of High-luminosity Spiral Galaxies. IV. Systematic Dynamical Properties, Sa through Sc. The Astrophysical Journal, 225, 107-111. https://doi.org/10.1086/182804

[14] Milgrom, M. (1983) A modification of the Newtonian Dynamics as a Possible Alternative to the Hidden Mass Hypothesis. The Astrophysical Journal, 270, 365-370. https://doi.org/10.1086/161130

[15] Weinberg, S. (1972) Gravitation and Cosmology: Principles and Application of the General Theory of Relativity. John Wiley and Sons, Hoboken, 619.

[16] Ray, S., Mukhopadhyay, U. and Ghosh, P.P. (2007) Large Number Hypothesis: a Review. https://arXiv.org/abs/0705.1836

[17] Guth, A.H. (1981) Inflationary Universe: A Possible Solution to the Horizon and Flatness Problems. Physical Review Letters D, 23, 347-356.

https://doi.org/10.1103/PhysRevD.23.347

[18] Rindler, W. (1956) Visual Horizons in World Models. Monthly Notices of the Royal Astronomical Society, 116, 662-677. https://doi.org/10.1093/mnras/116.6.662

[19] Dicke, R.H. and Peebles, P.J.E. (1979) The Big Bang Cosmology-Enigmas and Nostrums. In: Hawking, S. and Israel, W., Eds., General Relativity: An Einstein Centenary Survey, Cambridge University Press, Cambridge, 504-517.

[20] Peebles, P.J.E. and Ratra, B. (2003) The Cosmological Constant and Dark Energy. Review of Modern Physics, 75, 559-606. https://doi.org/10.1103/RevModPhys.75.559

[21] Trimble, V. (1987) Existence and Nature of Dark Matter in the Universe. Annual Review of Astronomy and Astrophysics, 25, 425-472. https://doi.org/10.1146/annurev.aa.25.090187.002233

[22] Corda, C. (2009) Interferometric Detection of Gravitational Waves: The Definitive Test for General Relativity. International Journal of Modern Physics D, 18, 2275-2282. https://doi.org/10.1142/S0218271809015904

[23] Duff, M.J. (2002) Comment on Time-Variation of Fundamental Constants. 
https://arXiv.org/abs/hep-th/0208093

[24] Duff, M.J., Okun, L.B. and Veneziano, G. (2002) Trialogue on the Number of Fundamental Constants. Journal of High Energy Physics, 3, 23.

https://doi.org/10.1088/1126-6708/2002/03/023 